\section{Osteogenic niche in the regulation of normal hematopoiesis and leukemogenesis}

\author{
Phuong M. Le, ${ }^{1}$ Michael Andreeff ${ }^{2}$ and Venkata Lokesh Battula ${ }^{2,3}$ \\ ${ }^{1}$ Department of Medical Oncology, Dana-Farber Cancer Institute, Boston, MA; ${ }^{2}$ Section \\ of Molecular Hematology and Therapy, Leukemia Department, The University of Texas \\ MD Anderson Cancer Center, Houston, TX and and ${ }^{3}$ Department of Breast Medical \\ Oncology, The University of Texas MD Anderson Cancer Center, Houston, TX, USA
}

\section{ABSTRACT}

T he bone marrow microenvironment, also known as the bone marrow niche, is a complex network of cell types and acellular factors that supports normal hematopoiesis. For many years, leukemia was believed to be caused by a series of genetic hits to hematopoietic stem and progenitor cells, which transform them to preleukemic, and eventually to leukemic, cells. Recent discoveries suggest that genetic alterations in bone marrow niche cells, particularly in osteogenic cells, may also cause myeloid leukemia in mouse models. The osteogenic niche, which consists of osteoprogenitors, preosteoblasts, mature osteoblasts, osteocytes and osteoclasts, has been shown to play a critical role in the maintenance and expansion of hematopoietic stem and progenitor cells as well as in their oncogenic transformation into leukemia stem/initiating cells. We have recently shown that acute myeloid leukemia cells induce osteogenic differentiation in mesenchymal stromal cells to gain a growth advantage. In this review, we discuss the role of the osteogenic niche in the maintenance of hematopoietic stem and progenitor cells, as well as in their transformation into leukemia cells. We also discuss the signaling pathways that regulate osteogenic nichehematopoietic stem and progenitor cells or osteogenic niche-leukemic stem/initiating cell interactions in the bone marrow, together with novel approaches for therapeutically targeting these interactions.

\section{Introduction}

Hematopoietic stem cells (HSCs) home to specific microenvironments in the bone marrow (BM) and receive signals that drive their fate under both normal and pathological conditions. So far, two predominant niches that differentially regulate HSCs through their non-hematopoietic compartments and levels of hypoxia have been identified. ${ }^{1,2}$ The endosteal niche near the inner bone surface is populated by osteoblastic lineage cells, including osteoprogenitor cells, pre-osteoblasts, mature osteoblasts, and osteocytes, as well as mesenchymal stromal cells (MSCs) and osteoclasts, whereas the non-endosteal niche consists mainly of sinusoidal endothelial cells, pericytes, and non-myelinating Schwann cells. Both niches are highly vascularized yet associated with distinct subtypes of blood vessels that support either the bone-forming or sinusoidal domain. ${ }^{3}$ Recent work from the Adams group also revealed a strong association between the osteogenic niche and a third vessel type that made up the transition zone in the developing bone. This subset seems to function upstream of both endosteal and sinusoidal endothelium, though more functionally related to the former, and connect the two vasculatures during the early stages of specialization. ${ }^{4}$ Stromal cells in both niches share overlapping signatures; however, it has been suggested that endosteal MSCs support HSC quiescence whereas non-endosteal MSCs promote HSC proliferation. ${ }^{5}$

Acute myeloid leukemia (AML) is one of the most aggressive hematologic malignancies, characterized by increased numbers of myeloid precursors in the $\mathrm{BM}$ that fail to differentiate into more mature myeloid cells. Recent studies have

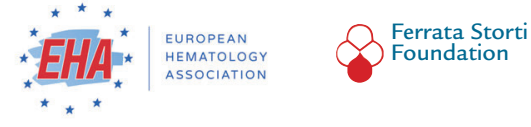

Haematologica 2018

Volume 103(12):1945-1955

\section{Correspondence:}

vbattula@mdanderson.org

Received: May 2, 2018.

Accepted: September 10, 2018.

Pre-published: October 18, 2018.

doi:10.3324/haematol.2018.197004

Check the online version for the most updated information on this article, online supplements, and information on authorship \& disclosures: www.haematologica.org/content/103/12/1945

(C)2018 Ferrata Storti Foundation

Material published in Haematologica is covered by copyright. All rights are reserved to the Ferrata Storti Foundation. Use of published material is allowed under the following terms and conditions:

https://creativecommons.org/licenses/by-nc/4.0/legalcode. Copies of published material are allowed for personal or internal use. Sharing published material for non-commercial purposes is subject to the following conditions:

https://creativecommons.org/licenses/by-nc/4.0/legalcode, sect. 3. Reproducing and sharing published material for commercial purposes is not allowed without permission in writing from the publisher. 
highlighted complex tumor-host interactions within the BM during AML progression. Malignant cells compete with their normal counterparts for niche resources and occupancy, and disrupt normal hematopoiesis by inflicting a differentiation block, which often manifests itself as BM failure and pancytopenia. ${ }^{67}$ In these conditions, leukemic cells seem to lose sensitivity to antiproliferative cues from the niche. ${ }^{8}$ Under the expansion of leukemia, MSCs have shown signs of "reprogramming" 9.11 In particular, the role of the osteoblast-rich region of the BM has been implicated in both AML chemoresistance and relapse. ${ }^{12,13}$ Unraveling the mechanisms underlying osteogenic niche-mediated support to AML cells is key to identifying molecular targets in order to develop effective drug therapies. In this review, we focus on advances in our understanding of the osteogenic niche in the leukemic BM microenvironment and discuss the key components of this niche as therapeutic candidates in AML.

\section{Osteolineage cells regulate normal hematopoiesis}

Non-random distribution of HSCs in the BM highlights the role of osteolineage cells in HSC maintenance. The physical association of HSCs with the endosteum correlates strongly with the colony formation and proliferative capacity of HSCs, and is primarily evident after BM transplantation..$^{14,15}$ Anatomical evidence has provided the basis on which the functional relationships between osteolineage cells and HSCs have continued to be unraveled. Osteoblasts secrete cytokines and growth factors including granulocyte-colony stimulating factor (G-CSF), ${ }^{16}$ hepatocyte growth factor, ${ }^{17}$ and osteopontin (OPN), ${ }^{18}$ which have been shown to maintain the pool size of the $\mathrm{CD} 34^{+}$progenitor population in the BM. Osteoblasts mediate HSC migration in and out of the BM, primarily through the CXCL12/CXCR $4^{19}$ and VCAM-1/NLA-4 ${ }^{20}$ axes, and under the influence of the sympathetic nervous system. ${ }^{21}$ In a knockout mouse model lacking bone morphogenetic protein (BMP) receptor I, Zhang et al..$^{22}$ reported that an increase in HSC number was associated exclusively with a cell population that lined the long bone and had an osteoblastic phenotype. Similarly, Calvi et al..$^{23}$ demonstrated that increasing osteoprogenitor or preosteoblast activation by augmenting parathyroid hormone (PTH) signaling enriched Lin- Sca-1+ ${ }^{+} \mathrm{c}-\mathrm{Kit}^{+}$, or HSClike, cells in vivo. Interestingly, this HSC expansion occurred without substantially affecting the overall number of hematopoietic cells. These observations suggest that PTH-induced signaling in osteoprogenitor cells or pre-osteoblasts might play a selective role in maintaining HSC self-renewal but not in the proliferation of their committed progenitors. How osteoblasts regulate HSC quiescence has been rigorously investigated. Loss of ligand-receptor interactions, such as angiopoietin-1 receptor tyrosine kinase 2 (Ang-1/Tie2) 24 and thrombopoietin-MPL (TPO/MPL), ${ }^{25}$ deregulates not only cell-cycle checkpoints but also coping mechanisms against extrinsic stressors, resulting in a reduction in slow-cycling hematopoietic cells. Stem-cell exhaustion and reduced self-renewal capacity after inhibition of Wingless (Wnt) signaling in osteoblasts further suggest that the mechanism underlying osteoblast-mediated regulation of HSCs does not follow a single axis. ${ }^{26}$

Surprisingly, osteoblast ablation, although associated with poorer HSC engraftment in vivo, does not lead to a massive loss of quiescent $\mathrm{HSCs}{ }^{27}$ It has also been shown that osteoblast deficiency in chronic inflammatory conditions, such as rheumatoid arthritis, does not affect the frequency of Lin- Sca-1+ ${ }^{+}$c-Kit ${ }^{+}$cells or their long-term repopulating potential. ${ }^{28}$ Mice with conditional deletion of CXCL12 $2^{29}$ or stem cell factor $(\mathrm{SCF})^{30}$ in osteoblasts do not exhibit HSC defects. It is possible that osteoblastic regulation of HSCs overlaps with other regulatory pathways and hence is easily compensated. Different osteolineage members may also share common signals while differing in the degree of impact. ${ }^{31}$ Together, these data suggest that osteolineage cells or more primitive cells such as MSCs orchestrate a diverse, though possibly non-essential, network of signals to maintain the stemness of HSCs and prompt hematopoietic activities, such as mobilization and expansion, in response to physiological needs.

\section{Altered osteogenic niche leads to myeloid leukemia in BM}

It has been firmly demonstrated that mutations affecting the ability of HSCs to differentiate into mature hematopoietic cells transform HSCs into pre-leukemic cells, and ultimately to leukemic cells when additional mutations are acquired (Figure 1). ${ }^{32-34}$ However, very little is known about the influence of other cellular components in the BM microenvironment on leukemic transformation of hematopoietic cells.

The Scadden group was the first to show that genetic alterations in osteolineage cells could lead to myelodysplastic syndromes (MDS) and leukemia. Deletion of Dicer1, a critical RNA processor and microRNA synthesizer, in Osterix (Osx)-expressing osteoprogenitor cells in a conditional knockout mouse model caused MDS and, on occasions, secondary AML. ${ }^{35}$ These mice first developed severe cytopenia and myelodysplasia, which transformed into monoblastic AML in 4 out of 200 cases, presenting as invasive myeloid sarcomas, anemia, and monocyte-like blast expansion in the peripheral blood, spleen, and BM. Of interest, Dicer 1 was intact in the myeloblastic tumors, suggesting that dysfunctional osteoblast precusors could mediate clonal evolution in neoplastic formation. Similarly, constitutive activation of $\beta$-catenin in mouse osteoblasts resulted in a broad spectrum of dysfunctional hematopoiesis, including monocytosis, lymphocytopenia, and somatic mutations that resembled those of human AML in myeloid progenitors. Kode et al.$^{36}$ noted that both wild-type mice engrafted with long-term (LT) HSCs from $\beta$-catenin-mutant mice and $\beta$-catenin-mutant mice engrafted with healthy BM cells developed AML and died shortly after transplantation. These observations suggest that an altered osteogenic niche could induce permanent damage to LT-HSCs and transform them to preleukemic and/or leukemic cells. Kousteni et al. attributed this niche-induced carcinogenesis to the oncogenic role of FoxO members involved in bone formation, which, surprisingly, are known tumor suppressors. ${ }^{37,38}$ This discovery sparks a debate about whether osteoblasts differentially regulate normal and malignant hematopoiesis. Recently, Dong et al..$^{39}$ also reported that mice with a mutant allele of protein tyrosine phosphatase SHP2 (Ptpn11) in osteoprogenitors or Nestin ${ }^{+}$MSCs could develop juvenile myelomonocytic leukemia-like myeloproliferative neoplasms (MPN). With concomitant mutations in HSCs, mice with mutated MSCs were twice as likely to progress from MPN to acute leukemia as were mice with altered endothelial cells. This study under- 
scores cell-type-specific leukemogenic effects of various niche components. While these findings in mice offer direct evidence for osteoblast-induced leukemogenesis, emerging reports of donor cell leukemia in humans (1-5\% of all post-transplant leukemia relapses), also suggest the role of an oncogenic microenvironment driving secondary malignancy. ${ }^{40}$ Collectively, it has been increasingly recognized that genetic aberrations in the endosteal compartment could be a key event in AML initiation and progression (Figure 1).

\section{AML induces osteogenic and osteolytic activity}

Numerous AML studies have emphasized the toxicity of leukemic expansion to BM niches. AML cells have been shown to alter BM niches by competing with HSCs for niche support, thereby affecting normal hematopoiesis. ${ }^{7,41,42}$ Whether the genomic landscape of non-hematopoietic components of BM niches changes has remained largely unexplored, and whether these alterations may drive AML initiation, progression, and resistance to chemotherapy is questionable.

Due to inconsistencies in methodology, cytogenetic analyses from different labs have led to a debate about the existence of chromosomal aberrations in leukemia patient BM-derived MSCs. ${ }^{10,43-45}$ To explore global changes induced by AML in stromal cells, our group performed a large-scale comparison of proteomic, microRNA, and gene expression profiles between AML patient-derived (AML-MSCs) and healthy donor-derived BM MSCs. We found upregulation of multiple pro-proliferative and anti-

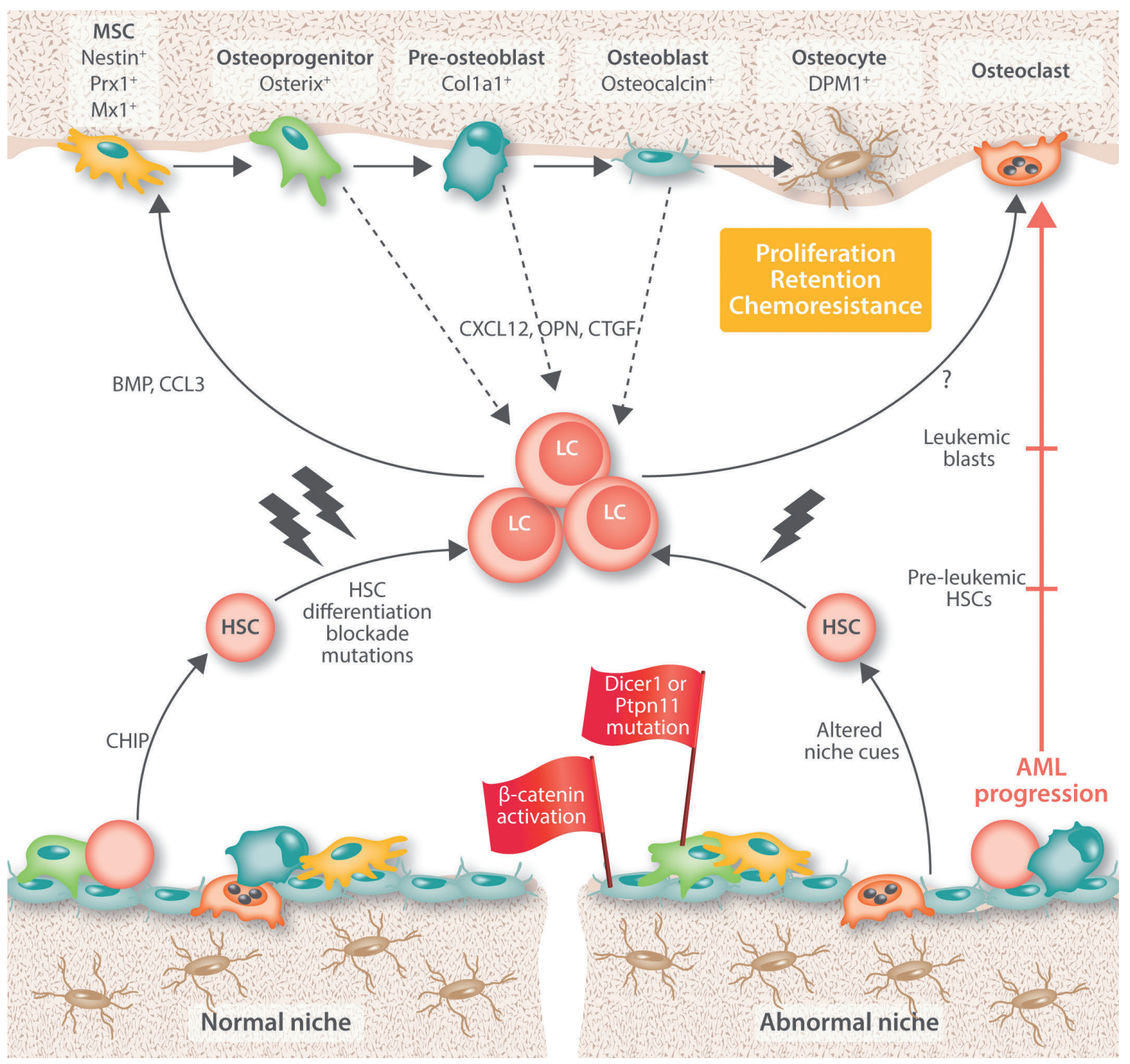

Figure 1. Osteogenic niche in hematopoietic stem cell (HSC) maintenance and leukemogenesis. Interactions between HSCs and osteogenic niche cells could happen in two ways. First, HSCs stay quiescent and self-renew when they are in osteogenic niche. When they acquire mutations under physiological stress, HSCs become pre-leukemic and eventually transform into leukemia blast cells. Alternatively, genetic abnormalities in osteogenic cells in the bone marrow could induce myeloid leukemia in non-mutated or in pre-leukemic HSCs. Second, leukemic cells could induce osteogenic differentiation in mesenchymal stromal cells (MSCs), which normally go through a series of differentiation steps to become fully mature osteoblasts or osteocytes. This feedback loop, involving bone remodeling, probably fuels leukemia progression. However, the extent to which acute myeloid leukemia (AML) cells induce osteogenic differentiation is not clear. BMP: bone morphogenetic protein; CHIP: clonal hematopoiesis of indeterminate potential; CTGF: connective tissue growth factor; HSC: hematopoietic stem cell; LC: AML cell; OPN: osteopontin. 
apoptotic pathways and downregulation of RNA regulators previously implicated in survival and differentiation of leukemic cells. ${ }^{46}$ Of particular interest, marked underexpression was observed for IGFBP5, an insulin-like growth factor binding protein that primarily inhibits osteoblast differentiation of MSCs. ${ }^{47}$ Moreover, TP53 was increased in AML-derived MSCs, resulting in senescence. We also found that the leukemia genotype, in particular the presence of FLT3-ITD mutations and lack of p53, induce both shared and leukemia genome-specific alterations in MSCs. ${ }^{48}$ These reports suggest that AML cells alter stromal development, and potentially their functionality.

Previously, Hanoun et al. had reported that AML primed MSCs to commit to osteoblastic lineage. ${ }^{49}$ The endosteal surface of mice transplanted with MLL-AFL9 leukemic cells was packed with Osx-expressing osteoprogenitor cells yet lacking Osteocalcin-positive $\left(O s c^{+}\right)$ mature osteoblasts. It is important to note that despite this osteogenic potential, these mice showed deficient bone mineralization and a lack of terminally differentiated osteoblasts compared with healthy controls. These observations were confirmed independently both in vitro and in vivo by our group. ${ }^{50}$ AML-MSCs displayed significantly higher alkaline phosphatase (ALP) expression and activity than did healthy donor-derived MSCs. In addition, when cultured in osteogenic differentiation medium, AML-MSCs differentiated to mature osteoblasts (alizarin red-positive) within two weeks compared with the three weeks needed for normal MSCs. Remarkably, gene expression analysis of normal MSCs co-cultured with different leukemic cell lines for five days revealed 2to 10-fold upregulation of osteogenic markers, such as Runt-related transcriptional factor (Runx2), Osx, Opn, and tissue non-specific ALP (Tnap), suggesting that this osteoprogenitor-priming pattern in MSCs resulted from AML exposure..$^{50}$ To validate AML-induced osteoblast differentiation in vivo, we created a human BM implant mouse model and assessed osteogenic potential of BM MSCs after four weeks of leukemia engraftment. Human MSCs obtained from these transplanted mice showed a 5- to 7fold increase in Osx and Runxz expression compared with control mice. ${ }^{50}$ These experimental data were consistent with OSX and RUNX2 upregulation in BM biopsies of AML patients.

We also found that AML-MSCs became less multipotent since they differentiated poorly into adipocytes and chondrocytes, two mesodermal lineages that usually arise from MSCs; the Bhatia group confirmed this adipocyte suppression in the setting of AML by immunostaining within human BM and global transcriptome analysis of AML-MSCs. ${ }^{51}$ Note that in the same study, gene sets poised towards osteoblast, but not adipocyte lineage were enriched in AML-MSCs, underscoring the need to understand the role of distinct mesenchymal fractions in AML progression.

We asked whether this osteolineage-specific priming provided any advantage for leukemic growth. Indeed, AML cells up-regulated connective tissue growth factor (CTGF) in MSCs and activated BMP signaling via Smad1/5 phosphorylation, both of which have been associated with persistence of tumors and poor prognosis in patients with acute leukemia. ${ }^{52-54}$ Besides, AML-induced TNAP overexpression in MSCs was implicated in osteoblast-mediated protection of leukemia blasts against apoptosis. ${ }^{55}$ By unraveling a feedback loop between stroma functionality and AML expansion, our study has highlighted the dynamics of the endosteal niche in AML pathogenesis (Figure 2).

The reduced bone mineralization seen by Hanoun et al. ${ }^{49}$ could have resulted from altered osteolytic activity. ${ }^{56}$ A short-lived increase in osteoclasts and upregulation of CCL3, a pro-inflammatory cytokine with pro-osteoclastic action previously established in multiple myeloma, ${ }^{57}$ was found in a murine model of blast-crisis chronic myeloid leukemia (CML) phenotype. ${ }^{56}$ These acute leukemia-like mice showed a significant reduction in $\mathrm{Osc}^{+}$osteoblasts and thinning of bone structures that could not be reversed completely by inhibition of osteoclasts. Bone deposition and resorption are tightly coupled processes that maintain bone homeostasis; however, this evidence suggests that the leukemic condition distorts this balance. Of interest, excessive CCL3 production does not typically lead to osteolytic lesions or bone loss, ${ }^{58}$ as seen in Ptpn11-mutated leukemic mice, ${ }^{39}$ although overexpression of this protein is common in the BM of AML patients. ${ }^{56}$ It is possible that monocyte differentiation into osteoclasts is defective in AML, yet the effects are masked by strong CCL3-driven recruitment of monocytes into the osteogenic niche..$^{59}$ The extent to which osteoblasts and osteoclasts work in tandem to reconstruct an inhospitable microenvironment under AML burden needs further investigation.

\section{Osteoprogenitors or mature osteoblasts: true 'partners-in-crime' in AML progression?}

The osteogenic niche comprises a variety of cell types which differ in their maturation status, ranging from very immature multipotent MSCs to mature osteoblasts and osteocytes (Figure 1). However, the differentiation status of osteogenic cells supporting normal hematopoiesis or leukemogenesis is an emerging question that remains to be resolved. Several studies have shown that, compared with less mature osteoblasts, terminally differentiated osteoblasts regulate HSC lineage commitment, such as B lymphopoiesis and erythropoiesis, while having less effect on HSC proliferation. ${ }^{60-62}$ Whether this functional stratification applies to the context of malignancy is poorly understood. Accumulating evidence has demonstrated that defective osteolineage cells are potent initiators of leukemia in the BM. These findings led to the question: which osteolineage cells, osteoprogenitors or mature osteoblasts, play a major role in promoting leukemogenesis?

As previously discussed, Raaijmakers et al. ${ }^{35}$ were the first to show that hematopoiesis could go awry as a result of a genetic alteration in osteoprogenitors. The Scadden group emphasized the differential leukemogenic capacity of immature and mature osteoblasts by comparing AML phenotype in Dicer $1^{\mathrm{f} / \mathrm{fl}}$ mice with Osx-versus Osc-driven Cre recombinase. Mice with a Dicer1 defect in mature osteoblasts did not exhibit any hematologic problem besides bone-related deformities. Similarly, Dong et al. ${ }^{39}$ confirmed the distinct role of stage-specific osteoblasts in leukemic development by generating mice with Ptpn11 mutations at various stages of MSCs: mesenchymal progenitor/stem cells, differentiated MSCs, Osx $x^{+}$osteoprogenitors, and $\mathrm{Osc}^{+}$mature osteoblasts. Of interest, the leukemogenic effect of this abnormality was observed in mice bearing the mutated form of either primitive MSCs 
or bone progenitor cells, but not more differentiated osteoblasts. These data are consistent with our observations that AML-MSCs show characteristics of osteoprogenitors but not of mature osteoblasts. AML-MSCs express early-stage osteoblast markers, including osterix, RUNX2, and Col1a1, but not mature osteoblast markers such as osteocalcin. ${ }^{50}$ In addition, functional assays revealed that AML-MSCs stained positive for ALP enzyme activity but were negative for alizarin red $S$ staining. ${ }^{50}$ These observations suggest that AML-MSCs can differentiate into committed osteoprogenitors, but not mature osteoblasts. These data were also validated by coculture of AML cell lines with normal BM-MSCs in vitro and by different AML mouse models. ${ }^{50,63}$ These findings also do not contradict the observations of Frisch et al., ${ }^{56}$ Geyh et al. ${ }^{64}$ and Krevvata et al. ${ }^{65}$ since the osteoblasts inhibited in these studies were marked by osteocalcin. Using intravital microscopy, Duarte et al. also showed a significant depletion of Col2.3 promoter-expressing mature osteoblasts in areas with a high level of AML filtration. ${ }^{66}$ Collectively, stalling the maturation of osteoblast precursors appears to be a key step in AML initiation and progression (Figure 2).

This differentiation blockade could be mediated by different AML-derived factors. Kumar et al. ${ }^{63}$ reported upregulation of DKK1, a negative regulator of osteogenesis, when co-culturing AML-derived exosomes with BM MSCs. Of particular interest, a short-term dose of DKK1

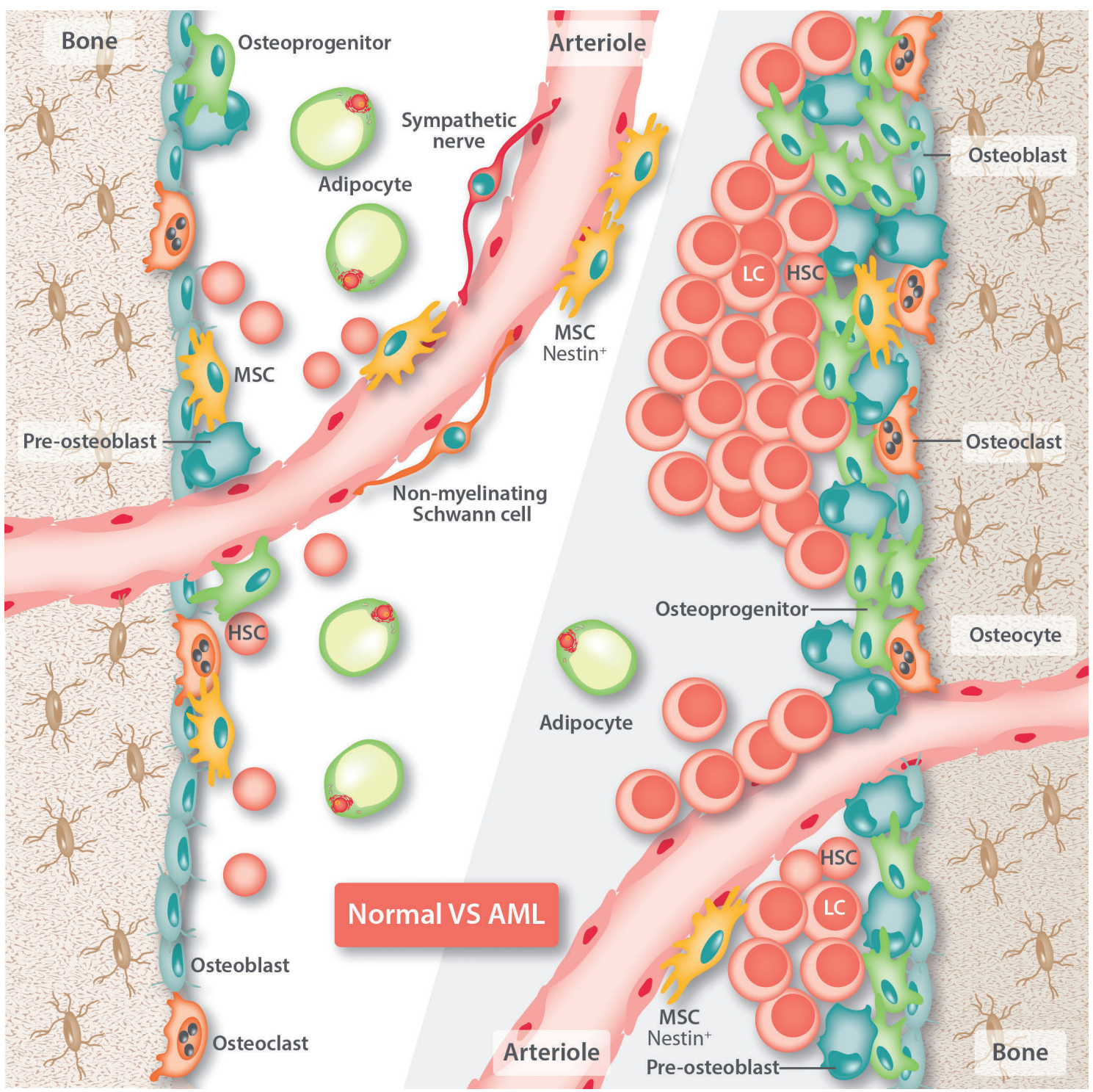

Figure 2. Schematic representation of normal versus acute myeloid leukemia (AML)-bone marrow (BM) microenvironment. Normal BM consists of osteoprogenitor cells, pre-osteoblasts, mature osteoblasts, and osteocytes, mesenchymal stromal cells (MSCs) and osteoclasts at endosteal niche and endothelial cells, pericytes, and non-myelinating Schwann cell at non-endosteal niche. In addition to these cell types, adipocytes are present throughout the BM cavity. Hematopoietic stem cells (HSC) are present in both niche areas and gain support from stromal cells to stay quiescent and self-renew, whereas in AML BM, leukemic blasts displace HSCs from the protective niche area and occupy this sanctuary, thereby affecting normal hematopoiesis. In addition, AML cells create or expand the existing niche by inducing osteogenic but inhibiting adipogenic differentiation in MSCs. However, there are no reports suggesting higher bone volume in AML patients. Therefore, it is possible that induction of osteogenic differentiation is halted at the osteo-progenitor or pre-osteoblastic stage. 
inhibitor promoted terminal differentiation of these osteolineage-primed MSCs in vitro and improved survival of mice engrafted with AML. This suggests a tight coupling of AML development with impaired maturation of osteoprogenitors. The fact that disruption of miRNA processing in immature, but not mature, osteoblasts could trigger AML development ${ }^{35}$ implies that deregulation of the maturation process at the post-transcriptional level might play a role in its failure. It would be interesting to further investigate the function of the miR-29 family, whose members are commonly down-regulated in AML blasts ${ }^{67}$ while engaging Wnt signaling antagonists, such as DKK1, in a feedback loop to promote osteolineage development. ${ }^{68}$ Another potential mediator is IL-1 $\beta$, a pleiotropic cytokine produced abundantly by AML blasts ${ }^{69}$ that has been shown to suppress osteogenesis of MSCs in periodontal tissue at a high physiological level. ${ }^{70}$ Whether one or more pathways are involved in causing this lack of osteoblast maturation remains to be elucidated.

Intriguingly, the effect of maturing osteoblasts on leukemia progression may be context-dependent and disease-specific. Schepers et al. ${ }^{71}$ showed that development of CML-like MPN induced by the BCR/ABL oncogene led to the expansion of a mixture of immature and mature osteoblasts that formed BM fibrosis and had decreased capacity to support HSCs. Krevvata et al. ${ }^{65}$ reported a strong correlation between the decrease in mature osteoblasts in mice and aggravated engraftment of different acute leukemia cell lines. In this study, over-stimulating $\mathrm{Osc}^{+}$osteoblast production by inhibiting gut-derived

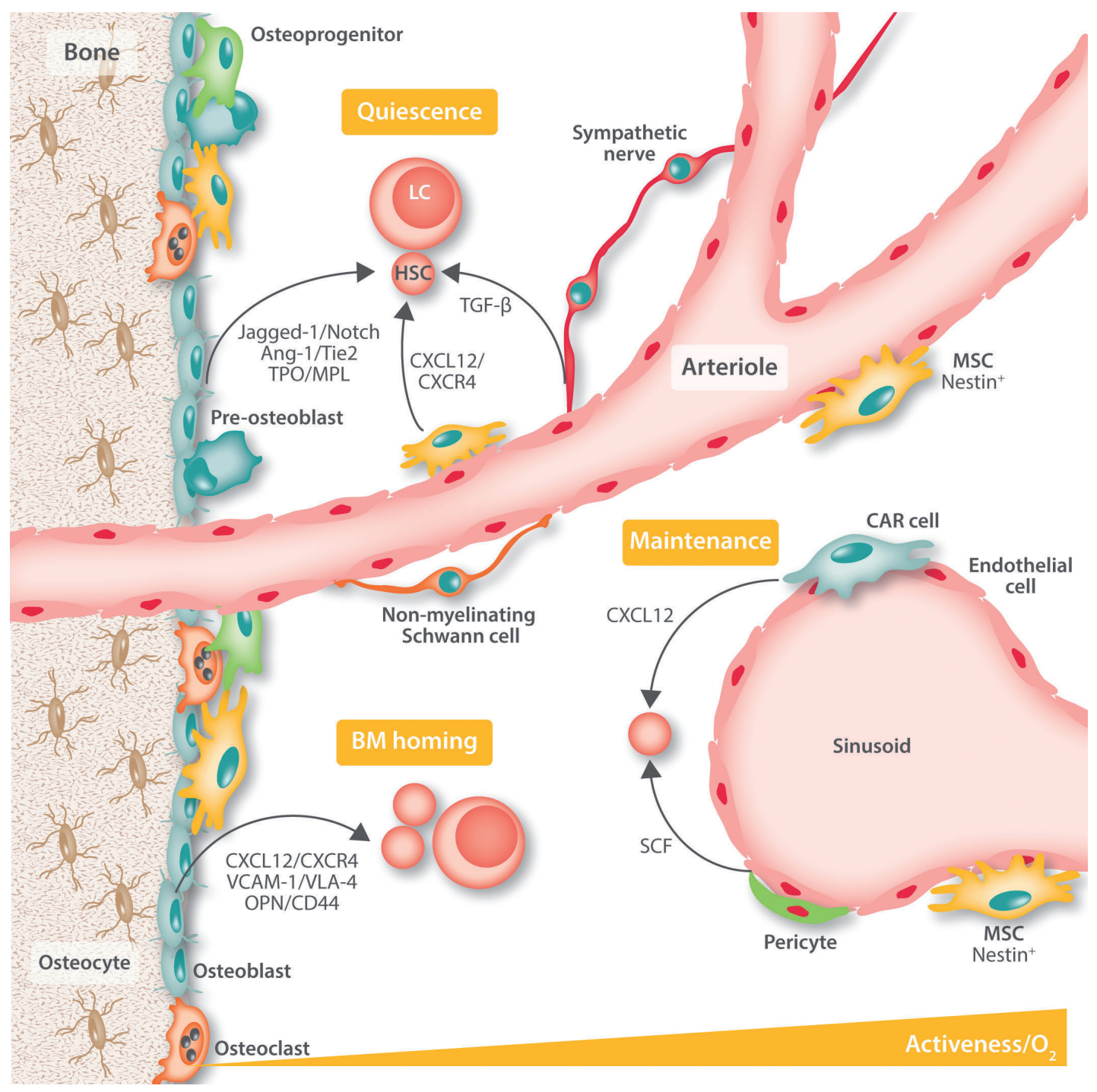

Figure 3. Key signaling pathways in the osteogenic niche that regulate the fate of hematopoietic stem cells (HSC) and leukemia stem/initiating cells (LSCs). HSCs home to bone marrow (BM) and receive maintenance signals from both endosteal and non-endosteal niches. Various cell types in the perisinusoid region, such as CXCL12-abundant reticular (CAR) cells and endothelial cells, maintain less quiescent HSCs via CXCL12 and stem cell factor (SCF). Non-myelinating Schwann cells and osteolineage cells, including mesenchymal stromal cells (MSCs), osteoprogenitors, and premature and mature osteoblasts, play a major role in retaining slowcycling HSCs near the bone surface. LSCs exploit the same cues from the osteogenic niche to hibernate and evade chemotherapy. In acute myeloid leukemia (AML), the BM niches are relatively hypoxic, whereas, in normal BM the hypoxic regions are more restricted to HSC-residing areas. Ang-1: angiopoietin 1; CXCL12: C-X-C motif chemokine 12; CXCR4: C-X-C chemokine receptor type 4; LSC: leukemic stem/initiating cell; OPN: osteopontin; SCF: stem cell factor; TGF- $\beta$ : transforming growth factor $\beta$; TPO: thrombopoietin; VCAM-1: vascular cell adhesion protein 1; VLA-4: very late antigen-4. 
serotonin synthesis in mice bearing MLL-AF9-induced AML attenuated disease burden. ${ }^{65}$ Conversely, using the same inducible model, Krause et al. ${ }^{8}$ showed that increasing osteoblastic activity through PTH activation augments leukemic expansion in AML while inhibiting CML-like MPN. ${ }^{8}$ These contrasting data are, at least in part, due to the heterogeneity of osteolineage cells that are characterized differently across studies. However, these results may also reflect the intrinsic differences in osteoblast-leukemia interaction between acute and chronic myeloid malignancies.

\section{Deregulated signaling network in the osteogenic niche offers promising therapeutic targets}

In the past decade, huge strides forward have been made in AML induction therapy by combining chemoand targeted therapies, yet this approach has offered limited success in preventing disease recurrence. It is assumed that the osteogenic niche shields slow-cycling AML cells from cell cycle-dependent treatment just as it maintains HSC quiescence (Figure 3); however, that is just the tip of the iceberg. The larger implication is that AML induces osteogenic dysfunction and disrupts the signaling network associated with the osteogenic niche (Table 1). This transformation of the niche could in turn fuel leukemia persistence and resistance to therapy. It is, therefore, critical to identify and target less visible threats underlying AML-osteogenic niche interactions to achieve more profound treatment efficacy.

Current approaches revolve mainly around disrupting BM homing axes, notably CXCL12/CXCR4. CXCR4 overexpression is common both at diagnosis ${ }^{72}$ and after chemotherapy, ${ }^{73}$ and correlates with poor prognosis in AML patients. ${ }^{74,75}$ CXCR4 inhibition prevents AML anchorage and promotes mobilization of leukemic stem/initiating cells (LSCs) out of the endosteal niche, thereby increasing their vulnerability to chemotherapy. Pre-clinical and clinical studies of CXCR4 antagonists have shown encouraging results, demonstrating that these agents not only sensitize AML cells to chemotherapy, but also reverse stroma-mediated antiapoptotic effects. ${ }^{76,77}$ Although the first generation of CXCR4

Table 1. Dysregulation of signaling network and potential therapeutic targets associated with osteogenic niche in acute myeloid leukemia (AML) and myeloproliferative neoplasms (MPN) progression.

\begin{tabular}{|c|c|c|c|c|c|}
\hline $\begin{array}{l}\text { Role in } \\
\text { normal } \\
\text { hematopoiesis }\end{array}$ & Pathway & $\begin{array}{l}\text { Deregulated } \\
\text { feature }\end{array}$ & $\begin{array}{l}\text { Prognostic } \\
\text { marker? }\end{array}$ & Contribution to leukemogenesis & Ref \\
\hline \multirow{3}{*}{$\begin{array}{l}\text { HSC homing } \\
\text { and retention in BM } \\
\text { osteogenic niche }\end{array}$} & CXCL12/CXCR4 & $\uparrow$ CXCR4 & Yes & $\begin{array}{l}\rightarrow \text { BM homing of AML LSCs } \\
\rightarrow \text { Prosurvival/antiapoptotic pathways }\end{array}$ & $\begin{array}{l}73-77 \\
81-84\end{array}$ \\
\hline & VCAM-1NLA-4 & ^ VLA-4 & Yes/No & $\begin{array}{l}\rightarrow \text { BM homing of AML LSCs } \\
\rightarrow \text { Stroma-mediated chemoresistance via NF-кB activation }\end{array}$ & $89-92$ \\
\hline & OPN/CD44 & $\begin{array}{l}\uparrow \mathrm{OPN} \\
\uparrow \mathrm{CD} 44 \mathrm{v}\end{array}$ & Yes & $\rightarrow$ BM homing of AML LSCs & 88,112 \\
\hline \multirow[t]{3}{*}{$\begin{array}{c}\text { HSC } \\
\text { maintenance }\end{array}$} & Jagged-1/Notch & $\begin{array}{l}\qquad \uparrow \uparrow \text { Jagged-1 } \\
\text { and Notch receptor } \\
\text { with limited autonomous } \\
\text { Notch activation }\end{array}$ & TBI & $\begin{array}{l}\text { Activation effect is context-dependent: } \\
\uparrow \downarrow \text { AML expansion in vitro } \\
\downarrow \text { AML progression (crosstalk unspecified) } \\
\uparrow \text { AML progression in crosstalk with } \beta \text {-catenin/FoXO1 } \\
\quad \text { in osteoblasts }\end{array}$ & $38,99,100,113$ \\
\hline & TGF- $\beta$ & $\begin{array}{l}\downarrow \text { Activation via } \\
\text { fusion gene }\end{array}$ & TBI & $\begin{array}{l}\text { Loss of TGF- } \beta \text { signaling is favorable though not required } \\
\text { for AML initiation. Activation effect is context-dependent: } \\
\rightarrow \text { LSC quiescence and chemoresistance } \\
\uparrow \text { AML progression \& } \downarrow \text { MPN progression via PTH } \\
\text { activation in osteoblasts } \\
\uparrow \text { AML progression via CTGF upregulation in MSCs }\end{array}$ & $8,11,50,114$ \\
\hline & BMP & $\begin{array}{l}\text { } A \text { Activation via fusion gene } \\
\text { Activation via osteogenic } \\
\text { priming by AML }\end{array}$ & Te TBI & $\uparrow$ AML progression in crosstalk with TGF- $\beta / C T G F$ in MSCs & 50,53 \\
\hline $\begin{array}{l}\text { Bone and } \\
\text { hematopoietic } \\
\text { homeostasis }\end{array}$ & SNS & Neuropathy & TBI & $\begin{array}{l}\text { } A M L \text { progression via osteogenic priming in MSCs } \\
\text { ^MPN progression via deficient sympathetic stimulation } \\
\text { of MSCs }\end{array}$ & 49,101 \\
\hline TBI & CCL3 & 个Proinflammatory cytokines & es TBI & MPN progression via HSC displacement & 39 \\
\hline
\end{tabular}

In AML cells; In osteolineage cells; $\uparrow$ Increase; $\downarrow$ Decrease; $\rightarrow$ Mediate; TBI: to be investigated; HSC: hematopoietic stem cells; CXCL12: stromal derived factor-1; CXCR4 C-X-C: chemokine receptor 4;VCAM-1: vascular cell adhesion protein-1;VLA-4: very late antigen-4; OPN: osteopontin; TGF- $\beta$ : transforming growth factor $\beta$; BMP:bone morphogenetic protein; SNS: sympathetic nervous system; CCL3: chemokine ligand 3; BM: bone marrow; LSC: leukemic stem/initiating cells; NF-kB: nuclear factor kappa-light-chain enhancer of activated B cells; PTH: parathyroid hormone; CTGF: connective tissue growth factor. 
inhibitors, such as AMD3100 (plerixafor) and AMD3465, show anti-leukemic effects only synergistically with chemotherapy and their action is rather transient, a second generation that has potential as monotherapy is emerging. ${ }^{78,79}$ Of note, the clinical benefit of CXCR4 blockade could be further optimized given the role of differentiating osteoblasts in shielding AML cells from CXCL12-mediated apoptosis in hypoxia. ${ }^{12}$ Though such induction of apoptosis ${ }^{80}$ is controversial, ${ }^{79,81-}$ ${ }^{83}$ it cannot be excluded that the interplay between hypoxia-mediated protection of leukemic cells typically found in AML BM ${ }^{84,85}$ and niche components may tip the balance between CXCL12-mediated pro-survival and apoptosis. This possibility is noteworthy given CXCR4 is a well-established target of HIF-1 $\alpha$, and therefore hypoxia. ${ }^{86}$ The role of HIF- $1 \alpha$ in survival and maintenance of CML has also been described. ${ }^{87}$ The Bonnet group found that HIF- $2 \alpha$, another factor that is regulated by hypoxia, plays a crucial role in regulation of the longterm re-populating ability of $\mathrm{CD} 34^{+}$umbilical cord blood cells. In addition, their data demonstrate that inhibition of HIF-2 $\alpha$ in primary AML cells inhibits their proliferation and sensitizes them to endoplasmic reticulum stress-induced apoptosis by upregulation of reactive oxygen species..$^{85}$

Similarly, chemosensitization and reduced AML engraftment could be achieved in mouse models with the use of CD44 specific antibody ${ }^{88}$ and VLA-4 blocking agents, such as natalizumab ${ }^{89}$ and AS101.90 Extramedullary BM models by Chen et al. ${ }^{91}$ and Jacamo et al..$^{92}$ demonstrated that AML and stromal cells interact via VLA-4 and VCAM1 to activate downstream NFkB signaling in both cell types. Blockade of these interactions resulted in inhibition of stroma-induced chemoresistance in AML cells. ${ }^{91,92}$ Besides, antagonizing these adhesion molecules has been found to relieve differentiation block in blasts, ${ }^{88}$ a clinical benefit also seen when epigenetic modifiers ${ }^{93}$ or FLT3 and IDH1/2 inhibitors ${ }^{94,95}$ were used to treat AML. This mobilizing approach, while preferentially mobilizing AML cells, carries the risk of moving HSCs out of their protective BM niche, among other adverse effects. ${ }^{96-98}$ Further randomized trials are needed to determine whether targeting AML homing axes is safe and how to optimize this chemosensitizing approach without impairing normal hematopoiesis.

There has been growing evidence to indicate another promising strategy: to target osteoblast function. Leukemia-stroma contact potentiates osteoblast differentiation in MSCs, which counteracts apoptogenic cues and promotes proliferative signals from the microenvironment to leukemic cells..$^{12,50,55}$ It has also been shown that abnormal signaling pathways and crosstalks that take place specifically in osteoblasts could induce or aggravate AML phenotype. eage cells would, therefore, render the osteogenic niche hostile to AML cells and abrogate the feedback loop fueling their perpetual life cycle. Indeed, modulation of mature osteoblast numbers by inhibiting gut-derived serotonin synthesis results in leukemia regression, providing a 'proof of concept' for this approach. ${ }^{65}$

The fact that stage-specific osteolineage cells have distinct functions and may differentially regulate normal and malignant hematopoiesis makes them an even more attractive target, especially with regard to their involvement in pleiotropic signaling pathways that support
HSCs, such as Notch or TGF- $\beta$. For example, despite already being known for its tumor-suppressor role in AML, ${ }^{99,100}$ Notch activation has been reported to be leukemogenic when synergizing with activating $\beta$ catenin/FoxO signaling in Colla $1^{+}$pre-osteoblasts. ${ }^{38}$ This observation suggests targeting FoxO signaling in preosteoblasts may be beneficial to patients with constitutive activating $\beta$-catenin mutation. As the effects of myeloid leukemia on cell differentiation along the osteolineage unfold, more leukemia modulators might be identified, and these will facilitate patient stratification and prevent treatment failure.

Restricting osteogenic capacity of MSCs could also be a therapeutic option. This strategy potentially limits the OPN reservoir of the BM, further preventing AML cells from hiding in the osteogenic niche and evading chemotherapy. Maintaining the primitive MSC pool via $\beta 2$ - and $\beta 3$-adrenergic agonists has shown multiple advantages in managing AML and MPN: rescuing healthy HSCs in the osteogenic niche with HSC maintenance factors and preventing LSCs from crowding out these normal residents. ${ }^{49,101}$ Studies have further shown that the bone surface and periarteriolar region are prone to inflammation during the early stage of osteogenic niche remodeling. ${ }^{39,56}$ This can be ameliorated by blocking receptors of pro-inflammatory cytokines, e.g. via CCL3 receptor antagonists.

Alternatively, promoting adipocyte differentiation of MSCs has been demonstrated to be a viable strategy to improve disease management by rescuing at least the generation of myeloid-erythroid lineages. ${ }^{51}$ However, the long-term efficacy of this pro-adipogenesis therapy remains to be tested given the debatable evidence about the role of adipocytes in AML seen so far. Different groups reported on adipocyte re-programming in which AML blasts exploit these energy reservoirs through lipolysis to fuel uncontrolled expansion. ${ }^{102-104}$ On the other hand, Lu et al ${ }^{105}$ only found a statistically significant correlation between AML patients' poor prognosis and an increase in small adipocytes, but not the decrease in largeor medium-sized ones. This finding suggests that lipid transfer may not be the only mechanism through which adipocytes aggravate leukemia burden. It cannot be excluded that, as previously shown in acute lymphoblastic leukemia, ${ }^{106,107}$ adipocytes may acquire a chemoprotective role in the setting of AML.

Recent discoveries provide evidence that mitochondria are transferred from BM stromal cells to leukemia cells which influence leukemia progression. ${ }^{108}$ These studies demonstrate that mitochondria are transferred via tunneling nanotubes (TNTs) or extracellular vesicles resulting in enhanced ATP production through increased oxidative phosphorylation (OXPHOS) which translates into higher drug resistance in AML cells and relapse after chemotherapy. ${ }^{109,110}$ Therefore, inhibition of mitochondrial transfer by targeting TNT formation or inhibiting OXPHOS is currently being considered as novel therapeutic strategies in AML therapy.

\section{Conclusions and emerging questions}

Findings on BM stroma-mediated chemoprotection in AML since the early 2000s have paved the way for a wave of new insights into leukemia-BM niche interactions, hence re-defining the paradigm of leukemic devel- 
opment and response to therapy. Although its fundamental role in HSC maintenance is still debatable, the osteogenic niche stands out as a pivotal sanctuary for LSCs and the cradle of blast production. Putting this into perspective, we foresee that AML-induced genetic changes and osteogenic priming in MSCs illustrate not only the long-standing multiple-hit hypothesis of carcinogenesis but also the newly-coined microenvironmentinduced oncogenesis. Importantly, the role of the BM niches in the development of MDS and AML from clonal hematopoiesis of indeterminate potential is still completely unknown. ${ }^{111}$ Many questions emerge, for instance, regarding the differential role of stage-specific osteolineage cells in AML progression, the uncoupling between osteogenesis and osteoclastogenesis, or the net therapeutic benefits of LSC dislodgement at the expense of HSC homelessness. Recent advances in our understanding of this osteoblast-rich region in AML progression provide a convincing premise with which to build the next genera- tion of AML therapy to target the osteogenic niche. However, further studies are needed to clarify the selfreinforcing loop between AML and the osteogenic niche, with the goal of inducing deep remissions and controlling long-term disease.

\section{Acknowledgements}

This work is supported by the Leukemia SPORE career development award (CA100632), Rolanette and Berdon Lawrence Research Award from Bone Disease Program of Texas, Institutional Research Grant (IRG) from MD Anderson Cancer Center, Cure Sonia Foundation and Golfers Against Cancer Foundation to VLB. In addition, this work was supported by grants from the National Institutes of Health (CA055164) and the MD Anderson Cancer Center Support Grant (CA016672), Cancer Prevention Research Institute of Texas (CPRIT, RP121010), and the Paul and Mary Haas Chair in Genetics to MA. We thank Dr. Marina Konopleva for her valuable suggestions in the preparation of this review article.

\section{References}

1. Morrison SJ, Scadden DT. The bone marrow niche for haematopoietic stem cells. Nature. 2014;505(7483):327-334.

2. Itkin T, Gur-Cohen S, Spencer JA, et al. Distinct bone marrow blood vessels differentially regulate haematopoiesis. Nature. 2016;532(7599):323-328.

3. Kusumbe AP, Ramasamy SK, Adams RH. Coupling of angiogenesis and osteogenesis by a specific vessel subtype in bone. Nature. 2014;507(7492):323-328.

4. Langen UH, Pitulescu ME, Kim JM, et al. Cell-matrix signals specify bone endothelial cells during developmental osteogenesis. Nat Cell Biol. 2017;19(3):189-201.

5. Asada N, Takeishi S, Frenette PS. Complexity of bone marrow hematopoietic stem cell niche. Int J Hematol. 2017:106(1):45-54.

6. Miraki-Moud F, Anjos-Afonso F, Hodby KA, et al. Acute myeloid leukemia does not deplete normal hematopoietic stem cells but induces cytopenias by impeding their differentiation. Proc Natl Acad Sci USA. 2013;110(33):13576-13581.

7. Glait-Santar C, Desmond R, Feng X, et al. Functional Niche Competition Between Normal Hematopoietic Stem and Progenitor Cells and Myeloid Leukemia Cells. Stem Cells. 2015;33(12):3635-3642.

8. Krause DS, Fulzele $K$, Catic A, et al. Differential regulation of myeloid leukemias by the bone marrow microenvironment. Nat Med. 2013;19(11):1513-1517.

9. Zhou HS, Carter BZ, Andreeff M. Bone marrow niche-mediated survival of leukemia stem cells in acute myeloid leukemia: Yin and Yang. Cancer Biol Med. 2016;13(2):248259.

10. Blau O, Hofmann W-K, Baldus CD et al. Chromosomal aberrations in bone marrow mesenchymal stroma cells from patients with myelodysplastic syndrome and acute myeloblastic leukemia. Exp Hematol. 2007;35(2):221-229.

11. Tabe Y Shi YX, Zeng Z et al. TGF-betaNeutralizing Antibody 1D11 Enhances Cytarabine-Induced Apoptosis in AML Cells in the Bone Marrow Microenvironment PLoS One. 2013;8(6):e62785.

12. Kremer KN, Dudakovic A, McGeeLawrence ME, et al. Osteoblasts Protect AML Cells from SDF-1-Induced Apoptosis. J Cell Biochem. 2014;115(6):1128-1137

13. Ishikawa F, Yoshida S, Saito $Y$, et al. Chemotherapy-resistant human AML stem cells home to and engraft within the bonemarrow endosteal region. Nat Biotechnol. 2007;25(11):1315-1321.

14. Lord BI, Testa NG, Hendry JH. The relative spatial distributions of CFUs and CFUc in the normal mouse femur. Blood. 1975;46(1):65-72.

15. Lo Celso C, Fleming HE, Wu JW, et al. Liveanimal tracking of individual haematopoiet ic stem/progenitor cells in their niche Nature. 2009;457(7225):92-96.

16. Taichman RS, Emerson SG. Human osteoblasts support hematopoiesis through the production of granulocyte colony-stimulating factor. J Exp Med. 1994;179(5):16771682.

17. Taichman RS, Reilly MJ, Verma RS, Ehrenman K, Emerson SG. Hepatocyte growth factor is secreted by osteoblasts and cooperatively permits the survival of haematopoietic progenitors. Br J Haematol. 2001;112(2):438-448.

18. Stier $\mathrm{S}, \mathrm{Ko} Y$, Forkert $\mathrm{R}$, et al. Osteopontin is a hematopoietic stem cell niche component that negatively regulates stem cell pool size. J Exp Med. 2005;201(11):1781-1791.

19. Sugiyama T, Kohara H, Noda M, Nagasawa T. Maintenance of the Hematopoietic Stem Cell Pool by CXCL12-CXCR4 Chemokine Signaling in Bone Marrow Stromal Cell Niches. Immunity. 2006;25(6):977-988.

20. Ulyanova T, Scott LM, Priestley GV, et al VCAM-1 expression in adult hematopoietic and nonhematopoietic cells is controlled by tissue-inductive signals and reflects their developmental origin. Blood. 2005;106(1): 86-94.

21. Katayama Y, Battista M, Kao WM, et al. Signals from the sympathetic nervous system regulate hematopoietic stem cell egress from bone marrow. Cell. 2006;124(2):407421.
22. Zhang J, Niu C, Ye L, et al. Identification of the haematopoietic stem cell niche and control of the niche size. Nature. 2003;425(6960):836-841

23. Calvi LM, Adams GB, Weibrecht KW, et al Osteoblastic cells regulate the haematopoietic stem cell niche. Nature. 2003;425 (6960):841-846

24. Arai F, Hirao A, Ohmura M, et al Tie2/angiopoietin-1 signaling regulates hematopoietic stem cell quiescence in the bone marrow niche. Cell. 2004;118(2):149 161.

25. Yoshihara H, Arai F, Hosokawa K, et al Thrombopoietin/MPL signaling regulates hematopoietic stem cell quiescence and interaction with the osteoblastic niche. Cell Stem Cell. 2007;1(6):685-697.

26. Fleming HE, Janzen V, Celso CL, et al. Wnt signaling in the niche enforces hematopoietic stem cell quiescence and is necessary to preserve self-renewal in vivo. Cell Stem Cell. 2008;2(3):274-283.

27. Bowers M, Zhang B, Ho Y, et al. Osteoblast ablation reduces normal long-term hematopoietic stem cell self-renewal but accelerates leukemia development. Blood. 2015;125(17):2678-2688

28. Ma YD, Park C, Zhao H, et al. Defects in osteoblast function but no changes in longterm repopulating potential of hematopoietic stem cells in a mouse chronic inflammatory arthritis model. Blood. 2009;114(20):44024410

29. Greenbaum A, Hsu Y-MS, Day RB, et al CXCL12 Production by Early Mesenchyma Progenitors is Required for Hematopoietic Stem Cell Maintenance. Nature. 2013;495(7440):227-230.

30. Ding L, Saunders TL, Enikolopov G, Morrison SJ. Endothelial and perivascular cells maintain haematopoietic stem cells Nature. 2012;481(7382):457-462

31. Nakamura Y, Arai F, Iwasaki $\mathrm{H}$, et al Isolation and characterization of endosteal niche cell populations that regulate hematopoietic stem cells. Blood. 2010;116 (9):1422-1432

32. Walter MJ, Shen D, Ding L, et al. Clonal Architecture of Secondary Acute Myeloid 
Leukemia. N Engl J Med. 2012;366(12):10901098.

33. Genomic and Epigenomic Landscapes of Adult De Novo Acute Myeloid Leukemia. N Engl J Med. 2013;368(22):2059-2074.

34. Shlush LI, Zandi S, Mitchell A, et al. Identification of pre-leukemic hematopoietic stem cells in acute leukemia. Nature. 2014;506(7488):328-333

35. Raaijmakers MH, Mukherjee S, Guo S, et al. Bone progenitor dysfunction induces myelodysplasia and secondary leukaemia. Nature. 2010;464(7290):852-857.

36. Kode A, Manavalan JS, Mosialou I, et al. Leukaemogenesis induced by an activating beta-catenin mutation in osteoblasts. Nature. 2014;506(7487):240-244.

37. Tothova Z, Gilliland DG. FoxO Transcription Factors and Stem Cell Homeostasis: Insights from the Hematopoietic System. Cell Stem Cell. 2007:1(2):140-152.

38. Kode A, Mosialou I, Manavalan SJ, et al. FoxO1-dependent induction of acute myeloid leukemia by osteoblasts in mice. Leukemia. 2016;30(1):1-13.

39. Dong L, Yu WM, Zheng $\mathrm{H}$, et al. Leukaemogenic effects of Ptpn11 activating mutations in the stem cell microenvironment. Nature. 2016;539(7628):304-308.

40. Wiseman DH. Donor Cell Leukemia: A Review. Biol of Blood and Marrow Transplantation. 2011;17(6):771-789.

41. Wang Y, Krivtsov AV, Sinha AU, et al. The Wnt/beta-catenin pathway is required for the development of leukemia stem cells in AML. Science. 2010;327(5973):1650-1653.

42. Lane SW, Wang YJ, Lo Celso C, et al. Differential niche and Wnt requirements during acute myeloid leukemia progression. Blood. 2011;118(10):2849-2856

43. Soenen-Cornu V, Tourino C, Bonnet M-L, et al. Mesenchymal cells generated from patients with myelodysplastic syndromes are devoid of chromosomal clonal markers and support short- and long-term hematopoiesis in vitro. Oncogene. 2005;24 (15):2441-2448

44. Huang JC, Basu SK, Zhao X, et al. Mesenchymal stromal cells derived from acute myeloid leukemia bone marrow exhibit aberrant cytogenetics and cytokine elaboration. Blood Cancer J. 2015;5:e302.

45. Blau O, Baldus CD, Hofmann WK, et al. Mesenchymal stromal cells of myelodysplastic syndrome and acute myeloid leukemia patients have distinct genetic abnormalities compared with leukemic blasts. Blood. 2011;118(20):5583-5592.

46. Kornblau SM, Ruvolo PP, Wang RY, et al. Distinct protein signatures of acute myeloid leukemia bone marrow-derived stromal cells are prognostic for patient survival. Haematologica. 2018;103(5):810-821.

47. Mukherjee A, Rotwein P. Insulin-Like Growth Factor-Binding Protein-5 Inhibits Osteoblast Differentiation and Skeletal Growth by Blocking Insulin-Like Growth Factor Actions. Mol Endocrinol. 2008;22 (5):1238-1250

48. Jacamo R, Davis RE, Ling X, et al. Tumor Trp53 status and genotype affect the bone marrow microenvironment in acute myeloid leukemia. Oncotarget. 2017;8(48): 83354-83369

49. Hanoun M, Zhang D, Mizoguchi T, et al. Acute myelogenous leukemia-induced sympathetic neuropathy promotes malignancy in an altered hematopoietic stem cell niche. Cell Stem Cell. 2014;15(3):365-375

50. Battula VL, Le PM, Sun JC, et al. AMLinduced osteogenic differentiation in mes- enchymal stromal cells supports leukemia growth. JCI Insight. 2017;2(13).

51. Boyd AL, Reid JC, Salci KR, et al. Acute myeloid leukaemia disrupts endogenous myelo-erythropoiesis by compromising the adipocyte bone marrow niche. Nat Cell Biol. 2017:19(11):1336-1347.

52. Sala-Torra O, Gundacker HM, Stirewalt DL, et al. Connective tissue growth factor (CTGF) expression and outcome in adult patients with acute lymphoblastic leukemia. Blood. 2007;109(7):3080-3083.

53. Crispino JD, Le Beau MM. BMP meets AML: induction of BMP signaling by a novel fusion gene promotes pediatric acute leukemia. Cancer Cell. 2012;22(5):567-568.

54. Raymond A, Liu B, Liang $\mathrm{H}$, et al. A role for BMP-induced homeobox gene MIXL1 in acute myelogenous leukemia and identification of type I BMP receptor as a potential target for therapy. Oncotarget. 2014;5(24): 12675-12693.

55. Sterner RM, Kremer KN, Dudakovic A, et al. Tissue-Nonspecific Alkaline Phosphatase Is Required for MC3T3 Osteoblast-Mediated Protection of Acute Myeloid Leukemia Cells from Apoptosis. J Immunol. 2018;201(3): 1086-1096.

56. Frisch BJ, Ashton JM, Xing L, et al. Functional inhibition of osteoblastic cells in an in vivo mouse model of myeloid leukemia. Blood. 2012;119(2):540-550.

57. Baba T, Mukaida N. Role of macrophage inflammatory protein (MIP)-1/CCL3 in leukemogenesis. Mol Cell Oncol. 2014;1(1): e29899.

58. Lane SW. Bad to the bone. Blood. 2012;119(2):323-325

59. Shi C, Pamer EG. Monocyte recruitment during infection and inflammation. Nat Rev Immunol. 2011;11(11):762-774.

60. Wu JY, Purton LE, Rodda SJ, et al. Osteoblastic regulation of B lymphopoiesis is mediated by $\mathrm{G}(\mathrm{s})$-dependent signaling pathways. Proc Natl Acad Sci USA. 2008:105(44):16976-16981.

61. Rankin EB, Wu C, Khatri R, et al. The HIF signaling pathway in osteoblasts directly modulates erythropoiesis through the production of EPO. Cell. 2012;149(1):63-74

62. Ding L, Morrison SJ. Haematopoietic stem cells and early lymphoid progenitors occupy distinct bone marrow niches. Nature. 2013;495(7440):231-235.

63. Kumar B, Garcia M, Weng L, et al. Acute myeloid leukemia transforms the bone marrow niche into a leukemia-permissive microenvironment through exosome secretion. Leukemia. 2017;32(3):575-587.

64. Geyh S, Rodriguez-Paredes M, Jager P, et al Functional inhibition of mesenchymal stromal cells in acute myeloid leukemia. Leukemia. 2016;30(3):683-691.

65. Krevvata M Silva BC, Manavalan IS, et al. Inhibition of leukemia cell engraftment and disease progression in mice by osteoblasts. Blood. 2014:124(18):2834-2846.

66. Duarte D, Hawkins ED, Akinduro O, et al. Inhibition of Endosteal Vascular Niche Remodeling Rescues Hematopoietic Stem Cell Loss in AML. Cell Stem Cell. 2017;22 (1):64-77.e6.

67. Gong JN, Yu J, Lin HS, et al. The role, mechanism and potentially therapeutic application of microRNA-29 family in acute myeloid leukemia. Cell Death Differ. 2014;21(1):100-112

68. Kapinas K, Kessler C, Ricks T, Gronowicz G, Delany AM. miR-29 Modulates Wnt Signaling in Human Osteoblasts through a Positive Feedback Loop. J Biol Chem. 2010;285(33):25221-25231.
69. Arranz L, Arriero MdM, Villatoro A Interleukin-1\&\#x3b2; as emerging therapeutic target in hematological malignancies and potentially in their complications. Blood Rev. 2017;31(5):306-317.

70. Mao Cy, Wang Yg, Zhang X, et al. Doubleedged-sword effect of IL-1 on the osteogenesis of periodontal ligament stem cells via crosstalk between the NF-B, MAPK and BMP/Smad signaling pathways. Cell Death Dis. 2016;7(7):e2296.

71. Schepers K, Pietras EM, Reynaud D, et al. Myeloproliferative Neoplasia Remodels the Endosteal Bone Marrow Niche into a SelfReinforcing Leukemic Niche. Cell Stem Cell. 2013;13(3):285-299.

72. Voermans C, van Heese WP, de Jong I, Gerritsen WR, van Der Schoot CE. Migratory behavior of leukemic cells from acute myeloid leukemia patients. Leukemia. 2002;16(4):650-657

73. Sison EAR, McIntyre E, Magoon D, Brown P. Dynamic chemotherapy-induced upregulation of surface CXCR4 expression as a mechanism of chemotherapy resistance in pediatric acute myeloid leukemia. Mol Cancer Res. 2013;11(9):1004-1016.

74. Konoplev S, Rassidakis GZ, Estey E, et al. Overexpression of CXCR4 predicts adverse overall and event-free survival in patients with unmutated FLT3 acute myeloid leukemia with normal karyotype. Cancer 2007;109(6):1152-1156.

75. Spoo AC, Lubbert M, Wierda WG, Burger JA. CXCR4 is a prognostic marker in acute myelogenous leukemia. Blood. 2007;109(2): 786-791.

76. Zeng Z, Shi YX, Samudio IJ, et al. Targeting the leukemia microenvironment by CXCR4 inhibition overcomes resistance to kinase inhibitors and chemotherapy in AML. Blood. 2009;113(24):6215-6224.

77. Nervi B, Ramirez P, Rettig MP, et al Chemosensitization of acute myeloid leukemia (AML) following mobilization by the CXCR4 antagonist AMD3100. Blood. 2009;113(24):6206-6214.

78. Kuhne MR, Mulvey T, Belanger B, et al BMS-936564/MDX-1338: A Fully Human Anti-CXCR4 Antibody Induces Apoptosis \&lt;em\&gt;In Vitro\&lt;/em\&gt; and Shows Antitumor Activity In Vivo in Hematologic Malignancies. Clin Cancer Res. 2013;19(2): 357-366.

79. Cho B-S, Zeng Z, Mu H, et al. Antileukemia activity of the novel peptidic CXCR4 antagonist LY2510924 as monotherapy and in combination with chemotherapy. Blood. 2015;126(2):222-232.

80. Kremer KN, Peterson KL, Schneider PA, et al. CXCR4 Chemokine Receptor Signaling Induces Apoptosis in Acute Myeloid Leukemia Cells via Regulation of the Bcl-2 Family Members Bcl-XL, Noxa, and Bak. J Biol Chem. 2013;288(32):22899-22914.

81. Zhang Y, Patel S, Abdelouahab H, et al. CXCR4 inhibitors selectively eliminate CXCR4-expressing human acute myeloid leukemia cells in NOG mouse model. Cell Death Dis. 2012:3(10):e396.

82. Borthakur G, Ofran Y, Nagler A, et al. The Peptidic CXCR4 Antagonist, BL-8040, Significantly Reduces Bone Marrow Immature Leukemia Progenitors By Inducing Differentiation, Apoptosis and Mobilization: Results of the Dose Escalation Clinical Trial in Acute Myeloid Leukemia. Blood. 2015;126(23):2546.

83. Chen Y, Jacamo R, Konopleva M, et al CXCR4 downregulation of let-7a drives chemoresistance in acute myeloid leukemia. J Clin Invest. 2013;123(6):2395-2407. 
84. Benito J, Ramirez M, Millward NZ, et al. Hypoxia-activated prodrug TH-302 targets hypoxic bone marrow niches in pre-clinical leukemia models. Clin Cancer Res. 2016;22(7):1687-1698.

85. Rouault-Pierre K, Lopez-Onieva L, Foster K, et al. HIF-2alpha protects human hematopoietic stem/progenitors and acute myeloid leukemic cells from apoptosis induced by endoplasmic reticulum stress. Cell Stem Cell. 2013;13(5):549-563.

86. Fiegl M, Samudio I, Clise-Dwyer K, et al. CXCR4 expression and biologic activity in acute myeloid leukemia are dependent on oxygen partial pressure. Blood. 2009;113(7): 1504-1512.

87. Zhang H, Li H, Xi HS, Li S. HIF1 is required for survival maintenance of chronic myeloid leukemia stem cells. Blood. 2012;119(11): 2595-2607.

88. Jin L, Hope KJ, Zhai Q, Smadja-Joffe F, Dick JE. Targeting of CD44 eradicates human acute myeloid leukemic stem cells. Nat Med. 2006;12(10):1167-1174.

89. Jiang E, Pham J, Kim H-N, et al. VLA4 Blockade In Acute Myeloid Leukemia. Blood. 2013;122(21):3944.

90. Layani-Bazar A, Skornick I, Berrebi A, et al. Redox Modulation of Adjacent Thiols in VLA-4 by AS101 Converts Myeloid Leukemia Cells from a Drug-Resistant to Drug-Sensitive State. Cancer Res J. 2014;74(11):3092-3103

91. Chen Y, Jacamo R, Shi YX, et al. Human extramedullary bone marrow in mice: a novel in vivo model of genetically controlled hematopoietic microenvironment. Blood. 2012;119(21):4971-4980.

92. Jacamo R, Chen Y, Wang Z, et al. Reciprocal leukemia-stroma VCAM-1/VLA-4-dependent activation of NF-kappaB mediates chemoresistance. Blood. 2014;123(17):26912702.

93. Abdel-Wahab O, Levine RL. Mutations in epigenetic modifiers in the pathogenesis and therapy of acute myeloid leukemia. Blood. 2013;121(18):3563-3572.
94. Sexauer A, Perl A, Yang X, et al. Terminal myeloid differentiation in vivo is induced by FLT3 inhibition in FLT3/ITD AML. Blood. 2012;120(20):4205-4214.

95. Boddu P, Borthakur G. Therapeutic targeting of isocitrate dehydrogenase mutant AML. Expert Opin Investig Drugs. 2017;26(5):525 530

96. Bonig H, Wundes A, Chang K-H, Lucas S, Papayannopoulou $\mathrm{T}$. Increased numbers of circulating hematopoietic stem/progenitor cells are chronically maintained in patients treated with the CD49d blocking antibody natalizumab. Blood. 2008;111(7):3439-3441.

97. Becker PS, Foran JM, Altman JK, et al. Targeting the CXCR4 Pathway: Safety, Tolerability and Clinical Activity of Ulocuplumab (BMS-936564), an AntiCXCR4 Antibody, in Relapsed/Refractory Acute Myeloid Leukemia. Blood. 2014;124 (21):386

98. Uy GL, Rettig MP, Stone RM, et al. A phase $1 / 2$ study of chemosensitization with plerixafor plus G-CSF in relapsed or refractory acute myeloid leukemia. Blood Cancer J. 2017;7(3):e542.

99. Lobry C, Ntziachristos P, Ndiaye-Lobry D, et al. Notch pathway activation targets AML-initiating cell homeostasis and differentiation. J Exp Med. 2013;210(2):301-319.

100. Klinakis A, Lobry C, Abdel-Wahab O, et al. A novel tumor suppressor function for the Notch pathway in myeloid leukemia. Nature. 2011:473(7346):230-233.

101. Arranz L, Sánchez-Aguilera A, Martín-Pérez $D$, et al. Neuropathy of haematopoietic stem cell niche is essential for myeloproliferative neoplasms. Nature. 2014;512(7512):78-81.

102. Tabe Y, Yamamoto S, Saitoh K, et al. Bone marrow adipocytes facilitate fatty acid oxidation activating AMPK and a transcriptional network supporting survival of acute monocytic leukemia cells. Cancer Res. 2017;77(6):1453-1464

103. Shafat MS, Oellerich T, Mohr S, et al. Leukemic blasts program bone marrow adipocytes to generate a pro-tumoral microenvironment. Blood. 2017;129(10): 1320-1332.

104. Battula VL, Chen Y, da Graca Cabreira M, et al. Connective tissue growth factor regulates adipocyte differentiation of mesenchyma stromal cells and facilitates leukemia bone marrow engraftment. Blood. 2013;122(3): 357-366.

105.Lu W, Weng W, Zhu Q, et al. Small bone marrow adipocytes predict poor prognosis in acute myeloid leukemia. Haematologica. 2018;103(1):e21-e24.

106. Behan JW, Yun JP, Proektor MP, et al. Adipocytes Impair Leukemia Treatment in Mice. Cancer Res J. 2009;69(19):7867-7874.

107. Sheng X, Tucci J, Parmentier J-H, et al Adipocytes cause leukemia cell resistance to daunorubicin via oxidative stress response. Oncotarget. 2016;7(45):73147-73159.

108. Griessinger E, Moschoi R, Biondani G Peyron JF. Mitochondrial Transfer in the Leukemia Microenvironment. Trends Cancer. 2017;3(12):828-839.

109. Moschoi R, Imbert V, Nebout M, et al. Protective mitochondrial transfer from bone marrow stromal cells to acute myeloid leukemic cells during chemotherapy. Blood. 2016;128(2):253-264

110. Marlein CR, Zaitseva L, Piddock RE, et al. NADPH oxidase-2 derived superoxide drives mitochondrial transfer from bone marrow stromal cells to leukemic blasts. Blood. 2017;130(14):1649-1660.

111. Steensma DP, Ebert BL. Clona Hematopoiesis after Induction Chemotherapy for Acute Myeloid Leukemia. N Eng J Med. 2018;378(13):1244-1245.

112.Liersch R, Gerss J, Schliemann C, et al Osteopontin is a prognostic factor for survival of acute myeloid leukemia patients. Blood. 2012;119(22):5215-5220

113. Tohda S, Nara N. Expression of Notchl and Jaggedl Proteins in Acute Myeloid Leukemia Cells. Leuk Lymphoma. 2001;42(3):467-472

114.Dong M, Blobe GC. Role of transforming growth factor- in hematologic malignancies. Blood. 2006;107(12):4589-4596 